\title{
A Rapidly Stabilizing Water-Gated Field-Effect Transistor Based on Printed Single-Walled Carbon Nanotubes for Biosensing Applications
}

\author{
Alireza Molazemhosseini, ${ }^{\dagger}$ Fabrizio Antonio Viola, ${ }^{\dagger}$ Felix J. Berger, ${ }^{\star}$ Nicolas F. Zorn ${ }^{\star}$ Jana \\ Zaumseil, ${ }^{*}$ Mario Caironi ${ }^{\dagger}, *$ \\ Center for Nano Science and Technology @PoliMi, Istituto Italiano di Tecnologia, Via \\ Giovanni Pascoli, 70/3, 20133 Milano, Italy \\ Institute for Physical Chemistry and Centre for Advanced Materials, Universitä̈ Heidelberg, \\ D-69120 Heidelberg, Germany \\ *Corresponding Author, Email: mario.caironi@,iit.it
}

\begin{abstract}
Biosensors are expected to revolutionize disease management through provision of low-cost diagnostic platforms for molecular and pathogenic detection with high sensitivity and short time-to-result. In this context, there has been an ever-increasing interest in using electrolytegated field-effect transistors (EG-FETs) for biosensing applications owing to their expanding potential of being employed for label-free detection of a broad range of biomarkers with high selectivity and sensitivity while operating at sub-volt working potentials. Whereas organic semiconductors have been widely utilized as the channel in EG-FETs primarily due to their compatibility with cost-effective low-temperature solution-processing fabrication techniques, alternative carbon-based platforms have the potential to provide similar advantages with improved electronic performances. Here we propose the use of inkjet-printed polymer-wrapped monochiral single-walled carbon nanotubes (s-SWCNT) for the channel in EG-FETs in an aqueous environment. In particular, we show that our EG-CNTFETs require only an hour of stabilization before producing a highly stable response suitable for biosensing, with a drastic time reduction with respect to the most exploited organic semiconductor for biosensors. As a proof-of-principle, we successfully employed our water-gated device to detect the well-known biotin-streptavidin binding event.
\end{abstract}

Keywords: electrolyte-gated; field-effect transistors; semiconducting carbon nanotubes; biosensors; bioelectronics; biotin; streptavidin 


\section{INTRODUCTION}

Recently, lot of efforts have been devoted to development of new classes of biosensors characterized by features such as a small form factor (i.e. handheld), low-manufacturing costs and fast time-to-response, aiming at point-of-care (POC) systems and self-testing platforms. Among different types of transducers used for biosensing, the field-effect transistor (FET) has become an attractive candidate due to its intrinsic signal amplification which results in a very high sensitivity. A variety of sensing platforms have been developed and extensively studied based on FETs such as ion-sensitive FETs, ${ }^{1}$ floating-gate FETs, ${ }^{2,3}$ and, more recently, electrolyte-gated FETs (EG-FETs), ${ }^{4}$ which have a remarkable potential, yet to be fully exploited. In a typical EG-FET, an ionically conducting and electronically insulating electrolyte is employed as gate insulator. Upon polarization of the gate, ions redistribution occurs, resulting typically in the formation of the so-called electric double layers (EDL), characterized by high capacitance values $\left(>1 \mu \mathrm{F} / \mathrm{cm}^{2}\right) .{ }^{5}$ As a consequence, EG-FETs are characterized by low operating voltages (less than $1 \mathrm{~V}$ ). Moreover, they do not require a reference electrode, which is needed, for instance, in ion-sensitive FETs, and offer label-free detection. In EG-FET biosensors, either the gate or the semiconductor can be biofunctionalized with a specific recognition element. ${ }^{6}$ Whereas introducing a bio-receptor into the semiconductor could turn to be challenging, gate functionalization has shown to be very robust and versatile thanks to high affinity of thiol-ending molecules to gold gates and to the experimental convenience of thiol functionalization procedures. ${ }^{7-9}$

The quest for low manufacturing costs of EG-FETs based biosensors has made solutionprocessed organic semiconductors a promising choice for the active material, ${ }^{10,11}$ given their compatibility with large-area and low-temperature printing techniques and with flexible, lowcost substrates. ${ }^{12-14}$ In organic electrolyte gated transistors, a distinction can be made between the case in which the semiconductor is not permeable to ions in the electrolyte, so that an EDL forms at this interface, and the case where a volumetric capacitance develops as an effect of ion permeation in the semiconductor thin film. In the latter case the device is historically addressed as organic electrochemical transistor (OECT) and exploits a volumetric field-effect. Despite the larger capacitance typically displayed by OECTs, the highest sensitivity has been reported so far for biosensors based on the former architecture, which typically goes under the name of electrolyte-gated organic field effect transistor (EGOFET). In fact, L. Torsi and collaborators recently reported poly(3-hexylthiophene) (P3HT) based EGOFET biosensors 
with a record sensitivity for millimetre sized devices, down to single-molecule (in the $\mathrm{zM}$ range), coupled to an excellent selectivity. ${ }^{4,15-17}$

The ultimate sensitivity reachable with biosensors based on solution-processed water gated polymer FETs and their compatibility with high-throughput manufacturing make the research toward real-world applicable printed EG-FETs biosensing platforms extremely appealing.

As a matter of fact, the large majority of water gated OFETs exploiting a solution-processed active layer make use of spin-coated films, while very few reports can be found on using printing techniques for deposition of the organic semiconductor channel. ${ }^{18,19}$

It is also a common trait of some of the most adopted organic semiconductors in water gated OFETs to undergo long stabilization times in water before producing a stable response suitable for biosensing. For instance, in case of P3HT EGOFETs, more than $12 \mathrm{~h}$ of water immersion are required. ${ }^{3,18,20-22}$ This process is attributed to a mild polymer swelling when is in direct contact with water, as demonstrated by the presence of hydroxyl moieties in the polymer film as well as its increased roughness after exposure to water. ${ }^{23} \mathrm{~A}$ similar behavior was also reported for EGOFETs that use organic semiconductors blended with polystyrene as their active layer. ${ }^{24}$ Indeed, such conditioning procedure imposes a constraint for the actual use of the biosensor.

In this respect, exploring alternative semiconductors that can be printed and share the same advantages of conjugated organic semiconductors in terms of manufacturing, while offering advantages in terms of electronic properties and stability under operation in water is very desirable. A powerful alternative is represented by semiconducting carbon nanotubes (CNTs). ${ }^{25-30}$ With recent advancement in sorting CNTs through selective polymer-wrapping technique, printable semiconducting single-walled carbon nanotubes (SWCNTs) formulations with high purity have become available. ${ }^{31,32}$ We have previously reported the successful adoption of spin-coated random networks of polymer-wrapped SWCNTs in electrolyte-gated carbon nanotubes FETs (EG-CNTFETs) for cell proliferation monitoring in aqueous cell cultures media. ${ }^{33}$ In this work, we present high performance EG-FETs operating in water based on inkjet-printed random networks of highly pure polymer-wrapped monochiral $(6,5) \mathrm{s}$ SWCNTs . Our EG-CNTFET shows a normalized transconductance $\left(g^{\prime} \mathrm{m}=g_{\mathrm{m}} \times L / W, L\right.$ being channel length and $W$ the channel width) of $0.44 \mu \mathrm{S}$, which is at least one order of magnitude higher compared to its EGOFET counterparts. ${ }^{4,24}$ Importantly, the device shows fast conditioning in water, producing a stable current after only one hour of consecutive acquisition of transfer curves, thus largely reducing the time constraints on its actual use as a sensor. In 
order to produce a proof-of-principle of its use as a base for future biosensors, we successfully adopted our inkjet-printed EG-CNTFET to characterize the well-known binding event of biotin to streptavidin. ${ }^{34-36}$ The inkjet-printed EG-CNTFET device presented in this study provides a stable and versatile platform that can be further exploited for development of arrays of printed biosensors.

\section{EXPERIMENTAL SECTION}

2.1. Preparation of s-SWCNT Dispersions. $(6,5) \mathrm{s}-\mathrm{SWCNT}$ dispersions were prepared from CoMoCAT® carbon nanotubes (Chasm Advanced Materials, SG65i-L58). According to the supplier, this CoMoCAT material has a maximum carbon content of $95 \%$, and $93 \%$ of which are SWCNTs. $40 \%$ of the nanotubes are $(6,5)$ SWCNTs, therefore $~ 35 \mathrm{wt} \%$ of the raw material. Shear force mixing (SFM) was used for sorting nanotubes through polymer-wrapping using poly[(9,9-dioctylfluorenyl-2,7-diyl)-alt-co-(6,6'-(2,2'-bipyridine)) $]$ (PFO-BPy). ${ }^{31}$ Briefly, 0.5 $\mathrm{g} / \mathrm{L}$ PFO-BPy (American Dye Source, $\mathrm{Mw}=40 \mathrm{~kg} / \mathrm{mol}$ ) were dissolved in toluene before adding $0.38 \mathrm{~g} / \mathrm{L}$ CoMoCAT raw material. SFM using a Silverson L2/Air mixer was then applied at maximum speed $(10,230 \mathrm{rpm})$ for $72 \mathrm{~h}$. The temperature was kept constant at $20^{\circ} \mathrm{C}$ with a cooling bath. Next, the dispersion was centrifuged twice for $45 \mathrm{~min}$ at $60,000 \mathrm{~g}$ and the supernatant was passed through a poly(tetrafluoroethylene) (PTFE) syringe filter (pore size $5 \mu \mathrm{m})$ to remove undispersed material and aggregates. To collect the SWCNTs, the resulting dispersion was filtered through a PTFE membrane (Merck Millipore, JVWP, $0.1 \mu \mathrm{m}$ pore size) and washed with toluene in order to remove excess polymer. The nanotubes ink was prepared through redispersion of the s-SWCNT filter cake in 1,2-dichlorobenzene by $30 \mathrm{~min}$ of bath sonication, obtaining an optical density of $1 \mathrm{~cm}^{-1}$ at the $\mathrm{E}_{11}$ transition.

2.2. FET Fabrication. Interdigitated source-drain electrodes with $W=4.4 \mathrm{~mm}$ and $L=3 \mu \mathrm{m}$ were patterned on a glass substrate by a mask-less reverse lithography process (AZ5214 photoresist together with MLA100 Heidelberg mask-less aligner) including thermal evaporation of chromium (2 $\mathrm{nm})$ and gold $(15 \mathrm{~nm})$, followed by lift-off in N-methyl-2pyrrolidone. Gold electrodes were subjected to $3 \mathrm{~min}$ of oxygen plasma treatment before inkjet printing. The SWCNTs ink was inkjet-printed (Fujifilm Dimatix, DMP2831) onto the active area of the patterned electrodes (an area of $300 \times 300 \mu \mathrm{m}^{2}$ ) with a drop-spacing of $60 \mu \mathrm{m}$. Printed patterns were annealed for $5 \mathrm{~min}$ at $200{ }^{\circ} \mathrm{C}$ in air and rinsed with tetrahydrofuran, isopropanol and dried with a nitrogen flux. The gate electrode was fabricated by thermally evaporating a $\mathrm{Cr}(2 \mathrm{~nm}) / \mathrm{Au}(50 \mathrm{~nm})$ stack on a polyethylene naphthalate $(\mathrm{PEN})$ sheet with a defined area of $3 \times 3 \mathrm{~mm}^{2}$. A film of inkjet-printed biocompatible insulator (SU8 - TF6001 
MicroChem) was employed to selectively passivate source and drain electrodes (Figure 1a), as well as to confine the sensing area of the gate electrodes adopted for biosensing.

2.3. Surface Bio-functionalization. Gold electrodes on PEN were rinsed in isopropanol for 5 min by bath sonication, followed by drying under a nitrogen flux. To form self-assembled monolayers of cysteamine (Cys-SAM), gold electrodes were immersed in a $100 \mathrm{mM}$ cysteamine (Sigma \#30070) solution prepared in 0.01 M phosphate buffer saline (PBS, Sigma Aldrich \#P4417) and kept in dark for $24 \mathrm{~h}$ inside a nitrogen glovebox. Subsequently, electrodes were rinsed with copious amount of DI water and were treated for $2 \mathrm{~h}$ with a freshly prepared $5 \mathrm{mg} / \mathrm{mL}$ solution of sulfosuccinimidyl-6-(biotinamido) hexanoate (Sigma Aldrich NHS-Xbiotin \#203189) in $0.01 \mathrm{M}$ PBS in air at room temperature (RT) by drop casting a $50 \mu \mathrm{L}$ of the solution onto the surface of the electrodes.

2.4. Electrical Characterization of EG-CNTFET. The electrical characterization of the EGCNTFET was performed by means of a Keysight B1500A Semiconductor Device Parameter Analyzer. The measurement setup included a Redox.me testing cell, which has a bottom magnet mount cell and a top glass reservoir for accommodating the electrolyte. The device was fixed in the cell and DI water was used as the gating medium. A standard $\mathrm{Ag} / \mathrm{AgCl}$ electrochemical electrode was employed as the gate for testing the device. The device was interrogated by consecutive recordings of transfer characteristic curves with a scan rate of $0.025 \mathrm{~V} / \mathrm{s}$ for $4 \mathrm{~h}$ with an interval of $3 \mathrm{~min}$ in between the acquisitions, during which the device was at tri-state. Each curve acquisition lasts for $80 \mathrm{~s}$.

2.5. Biosensing Measurement. For the proof-of-principle of biosensing experiment, the same setup used for electrical characterization of the EG-CNTFET was used, adopting the $\mathrm{Ag} / \mathrm{AgCl}$ electrode as the control gate (C-gate) to monitor the stability of the transistor, while a functionalized gold gate (F-gate) was immersed in the water reservoir and exploited for biosensing tests (Figure S1). Before a biosensing experiment, the device undergoes a conditioning procedure to reach electrical stability. This stability protocol involves interrogation of the device kept in water by consecutive recordings of transfer characteristic curves with 3 min intervals, until the threshold voltage $\left(V_{\text {th }}\right)$ and the current of the device stabilize. The protocol considers $V_{\text {th }}$ stable when the rate of variation is equal or lower than 1 $\mathrm{mV} / \mathrm{h}$ and considers the current stable when the rate of the ratio between its temporal variation $(\Delta I)$ and its initial value $\left(I_{0}\right)$ reaches $0.002 \mathrm{~h}^{-1}$. C-gate is used to apply the stabilization protocol. The last transfer curve obtained at the end of the stabilization protocol is used as the reference to be compared with a transfer curve acquired on C-gate immediately after obtaining the 
calibration curve. This is to validate the device stability during the biosensing measurement. Standard solutions of streptavidin were prepared with concentrations ranging from $1.6 \mathrm{nM}$ to 1.6 $\mu \mathrm{M}$ in $0.01 \mathrm{M}$ PBS for the purpose of acquisition of the calibration curve. Negative control measurements were performed by substituting streptavidin with bovine serum albumin (BSA). The titration curve was acquired by drop casting different concentrations of streptavidin solutions on F-gates and incubating for $20 \mathrm{~min}$ at RT. F-gates were then rinsed with DI water and immersed in DI water for 10 min before being mounted one by one on the device to record the transfer response. For each concentration of streptavidin, three physically different F-gates were measured.

2.6. s-SWCNT Ink and Film Characterization. Optical characterization of s-SWCNT ink was performed by recording absorption spectra of the formulation using a PerkinElmer Lambda 1050 spectrometer. The surface topography of the s-SWCNT inkjet-printed random network was measured with an Agilent 5500 atomic force microscope operated in tapping mode.

\section{RESULTS AND DISCUSSION}

3.1. EG-CNTFET electrical properties. First, we aimed at assessing the electrical performance of EG-FETs based on printed random networks of polymer-wrapped monochiral $(6,5) \mathrm{s}-\mathrm{SWCNTs}$. The relatively large bandgap of $(6,5) \mathrm{s}-\mathrm{SWCNTs}(\approx 1.27 \mathrm{eV})$ compared to semiconducting nanotubes with other chiralities is reported to contribute to low off current in field-effect transistors. ${ }^{37}$ The sorting process through selective wrapping with PFO-BPy involves shear force mixing, which is a mild dispersion method compared to other techniques such as sonication and introduces less structural damage to the SWCNTs. ${ }^{31}$ Consequently, a larger average length of nanotubes can improve the charge transport properties of a random network. Our EG-CNTFET is fabricated by inkjet-printing of the nanotubes onto interdigitated gold electrodes after brief oxygen plasma treatment. Figure 1a presents an optical microscope image of the active area of the device, showing the interdigitated electrodes and the contact leads passivated with inkjet printed SU8 in order to prevent their exposure to the electrolyte. Nanotubes printed area, which is not visible under optical microscope, is indicated with a red box in Figure 1a. The absorption spectrum of the s-SWCNTs ink prepared for printing (Figure 1b) evidences the two main absorption peaks $\left(E_{11}\right.$ and $\left.E_{22}\right)$ of $(6,5)$ nanotubes at $575 \mathrm{~nm}$ and at $996 \mathrm{~nm}$, along with the PFO-BPy absorption signature at shorter wavelengths. The ink absorbance indicates absence of metallic nanotubes within the detection limit and a high degree of monochirality. AFM topography of the inkjet-printed s-SWCNTs formulation (Figure 1c) 
evidences a dense random network of nanotubes. Although a single carbon nanotube (average length of about $1.82 \mu \mathrm{m}^{31}$ ) is not long enough to bridge the $3 \mu \mathrm{m}$ channel gap of the device, the random network allows a charge percolation through multiple pathways between source and drain.

For electrical characterization, the device is allocated in a testing cell (Figure 1d) and is immersed in DI water acting as gating medium. To obtain the transfer characteristic curves of the EG-CNTFET, an $\mathrm{Ag} / \mathrm{AgCl}$ electrode immersed in DI water is used as gate, exploiting its well-known stability in aqueous environments. A typical transfer characteristic curve of the printed EG-CNTFET (Figure 1e) shows good p-type operation, with a channel current reaching $18 \mu \mathrm{A}$ at a gate voltage $\left(V_{\mathrm{g}}\right)$ of $-0.8 \mathrm{~V}$, a current on/off ratio higher than $10^{3}$, and a very low gate leakage current, 5 orders of magnitude lower than the on current (at $V_{\mathrm{g}}=-0.8 \mathrm{~V}$ ). The device achieves a maximum transconductance $g_{\mathrm{m}}$ of $\sim 645 \mu \mathrm{s}$, corresponding to a normalized transconductance $\left(g_{\mathrm{m}}\right.$ divided by $\left.W / L\right)$ of $0.44 \mu \mathrm{S}$ (Figure $\left.1 \mathrm{f}\right)$. Overall, our printed EGCNTFET demonstrates a current on/off ratio comparable to the best water-gated EGOFET counterparts, ${ }^{4,19}$ while showing at least one order of magnitude higher normalized transconductance. ${ }^{11,38}$ Therefore, the proposed water-gated device is both compatible with large-area printing processes and delivers competitive electrical performances.

a

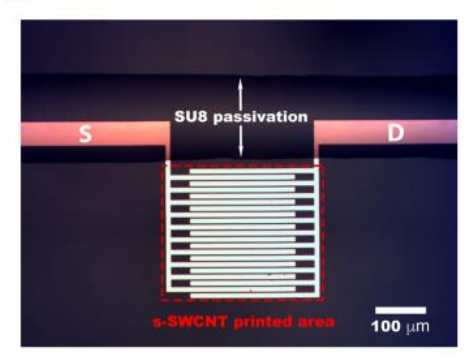

d

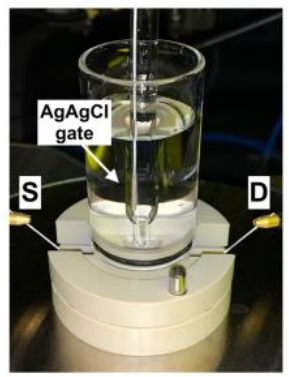

b

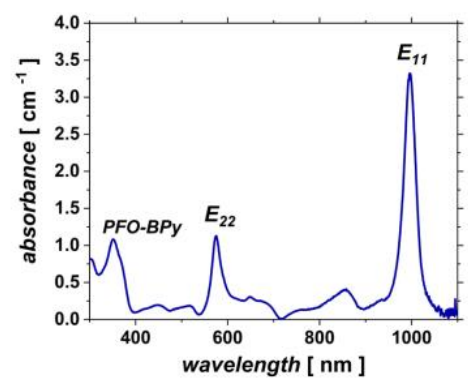

e

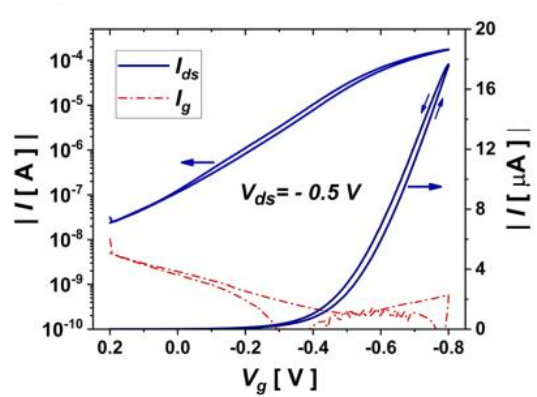

C

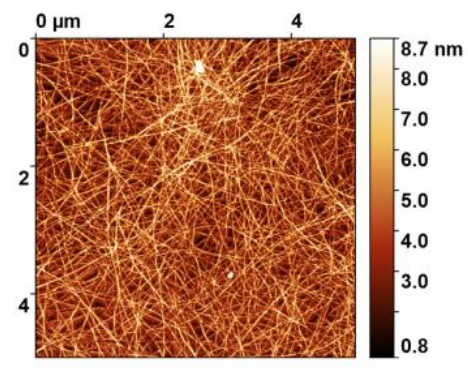

f

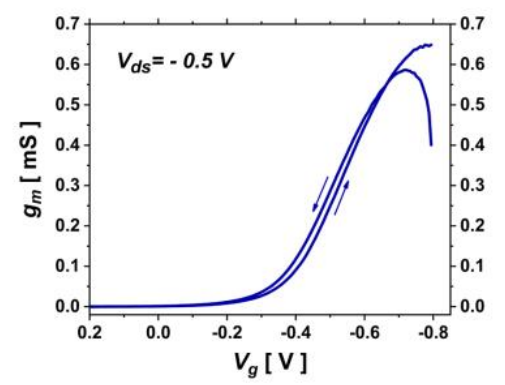

Figure 1. (a) Optical microscope image of the sensing area of the device showing the printed s-SWCNT area (dashed red rectangular, nanotubes are not visible under optical microscope) onto the interdigitated electrodes and passivated contacts with printed SU8. (b) Absorption spectrum of the (6,5) s-SWCNT ink prepared for printing. (c) AFM topography of a typical ink-jet printed random network of nanotubes. (d) The testing setup used for electrical characterization of the device, consisting of a glass reservoir for 
accommodating the electrolyte, water in this case, a magnet mount cell at the bottom to sandwich the chip with a circular opening (diameter of $6 \mathrm{~mm}$ ) to expose the device to the electrolyte, and an $\mathrm{Ag} / \mathrm{AgCl}$ reference electrode immersed in the electrolyte, used as gate. (e) Typical transfer characteristic curve of EG-CNTFET in semilogarithmic (left axis) and linear (right axis) scales showing the drain current in blue together with the gate leakage current in dashed red, which is five orders of magnitude lower than drain current at $V_{\mathrm{g}}=-0.8 \mathrm{~V}$.(f) Transconductance of the EG-CNTFET calculated from the transfer curve in (e).

3.2. Stabilization of the EG-CNTFET in water. A stable device response upon exposure to the electrolyte is crucial for biosensing applications. No sensitive biosensing is possible if the device current does not reach a suitably stable value allowing to discern variations induced by the biorecognition event with respect to inherent fluctuations in the device current. Consequently, a stabilization protocol must be designed and followed prior to biosensing tests. The stability of the proposed device when operating in water was evaluated using an $\mathrm{Ag} / \mathrm{AgCl}$ electrode as gate (Figure 1d), by measuring a series of consecutive transfer characteristic curves with 3 min intervals for the duration of $4 \mathrm{~h}$ (Figure $2 \mathrm{a}$ ). In order to quantify any variation in device response during the test, the threshold voltage and the normalized channel current change at fixed potential $\left(\Delta I / I_{0}\right.$ at $\left.V_{\mathrm{g}}=-0.8 \mathrm{~V}\right)$ were extracted from the transfer characteristic curves acquired at different time intervals. According to Figure 2a, the transfer curve undergoes the most evident change in the first $\sim 10 \mathrm{~min}$, i.e. within the first three transfer cycles. The extracted threshold voltages (black triangle symbol in Figure 2b) reveal that after an hour of acquisition of consecutive transfer curves, the device stabilizes with a very weak residual shift of its threshold voltage of $\approx 1 \mathrm{mV} / \mathrm{h}$ toward negative potentials. For the purpose of comparison, an optimized inkjet-printed P3HT based EGOFET was fabricated (details in Supplementary Information) and subjected to the same transfer cycling test, at the same biasing conditions, in DI water. As shown in Figure 2b, the threshold voltage of P3HT based EGOFET (black square symbol) does not stabilizes after $4 \mathrm{~h}$ of transfer cycling. Correspondingly, the normalized current change for EG-CNTFET (Figure 2b, red triangle symbol) shows a $13.8 \%$ decrease during the first hour of transfer cycling, before showing a substantial stabilization, with a residual very weak and constant decay of $0.002 \mathrm{~h}^{-1}$ until the end of the fourth hour. The P3HTEGOFET (Figure 2b, red square symbols) shows $26.4 \%$ decrease in normalized current change after an hour of acquisition of consecutive transfer characteristic curves. Then the current decay exacerbates without a constant rate until the end of the fourth hour, reaching a reduction of more than $60 \%$ and indicating that the device needs a longer conditioning procedure before showing a stable response. In fact, in agreement with previous literature, ${ }^{18}$ the inkjet-printed 
P3HT-EGOFET needs $30 \mathrm{~h}$ of transfer cycling before producing a stable current response with a $\Delta I / I_{0}$ change rate of $0.002 \mathrm{~h}^{-1}$ (Figure $\left.\mathrm{S} 2\right)$.

Overall, acquisition of consecutive transfer characteristic curves shows that an hour of transfer cycling in DI water is enough to stabilize the I-V response of the EG-CNTFET, with a negligible residual threshold voltage shift of $1 \mathrm{mV} / \mathrm{h}$ and $\Delta I / I_{0}$ decay rate of $0.002 \mathrm{~h}^{-1}$. This evidence shows that the device can be potentially employed for biosensing after only an hour of pre-conditioning and is stable for at least 3 more hours.

a

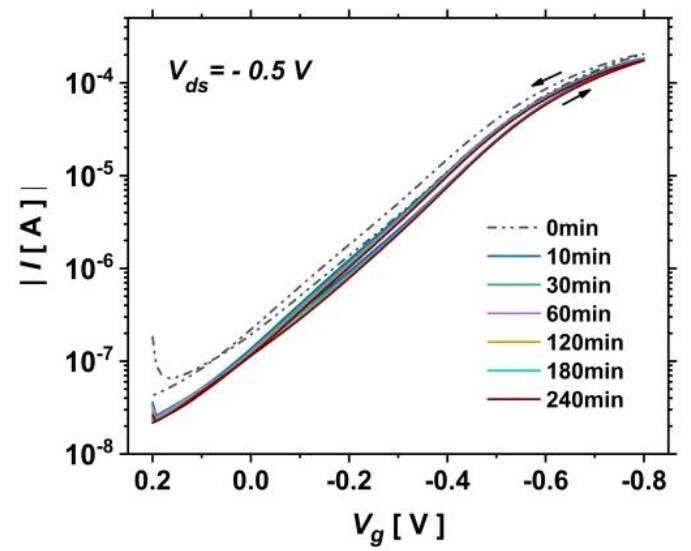

b

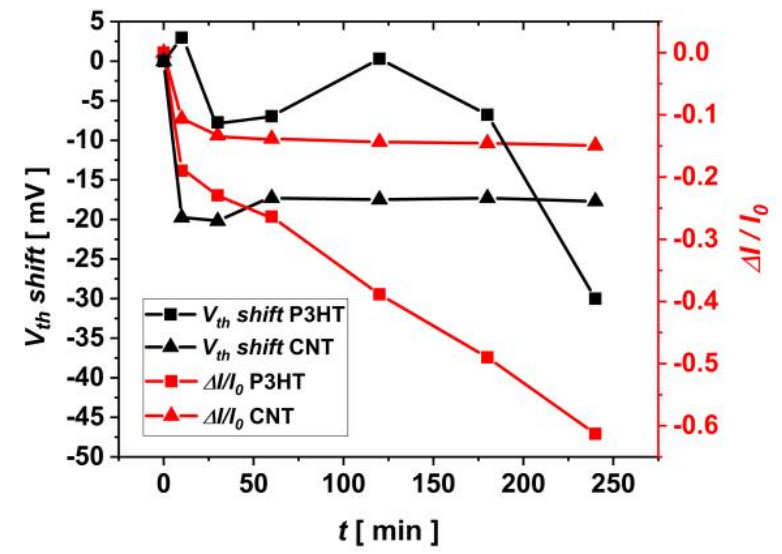

Figure 2. (a) Forward and backward transfer curves cycling measurements of the EG-CNTFET in DI water using an $\mathrm{Ag} / \mathrm{AgCl}$ gate for a total duration of $4 \mathrm{~h}$, with an interval of 3 min between successive curves; each curve acquisition lasts for $80 \mathrm{~s}$. (b) Threshold voltage shift (left vertical axis) and normalized current change at $-0.8 \mathrm{~V}$ (right vertical axis) for EG-CNTFET (triangle symbol) and P3HTEGOFET (square symbol) during $4 \mathrm{~h}$ of transfer cycling with 3 min interval. Negative sign of the $V_{t h}$ shift signifies a shift toward negative potentials.

3.3. Biosensing test. To assess the suitability of our EG-CNTFET as the base of a biosensor, we performed a proof-of-principle experiment aimed at sensing the biotin-streptavidin binding event. For this purpose, a gold electrode is introduced, acting as the sensing gate (F-gate): such gate is biofunctionalized to accommodate a biotin-terminated recognition layer. Apart from Fgate used for biosensing, an $\mathrm{Ag} / \mathrm{AgCl}$ reference gate, denoted as $\mathrm{C}$-gate, is utilized to monitor the stability of the device during biosensing measurement.

A schematic illustration of F-gate bio-functionalization is shown in Figure 3a. In detail, a selfassembled monolayer (SAM) of cysteamine (Cys-SAM) is grown on the gold electrode. Then, treatment of Cys-SAM terminated gold electrodes with NHS-LC-biotin molecule results in substitution of NHS group with the amine group of Cys-SAM. As a result, a molecular bioreceptor layer forms, anchored to the gold electrode, through a thiol group, at one end exposing a biotin moiety at the other end (biotin-SAM). The biosensing experiment involves 
the exposure of the biotin functionalized F-gates to standard solutions of streptavidin, with concentration varying from $1.6 \mathrm{nM}$ to $1.6 \mu \mathrm{M}$. The binding of streptavidin to the biotin-SAM is then sensed by using the exposed F-gates as gating electrodes of the EG-CNTFET. It should be noted that pure water is a weak electrolyte, following self-ionization $\left(\left[\mathrm{H}_{3} \mathrm{O}^{+}\right]=\left[\mathrm{OH}^{-}\right]=10^{-7}\right.$ M) and dissolution of carbon dioxide. ${ }^{39}$ Therefore its Debye length is quite long, typically 100 $\mathrm{nm},{ }^{4}$ well above the distance at which the binding occurs considering the theoretical length of the molecular receptor which is about $3 \mathrm{~nm}$.

As shown in Figure 3b, exposure of the bio-functionalized F-gate to increasing concentrations of streptavidin leads to recording a correspondingly increasing negative shift in the threshold voltage. Figure $3 \mathrm{c}$ presents the titration curve obtained considering the device threshold voltage shift extracted at $V_{\mathrm{g}}=-0.5 \mathrm{~V}$ from the transfer curves obtained for different concentrations of the target protein. Comparing the lowest threshold voltage shift recorded in our experiment for streptavidin-captured F-gates (13.75 $\mathrm{mV}$ for $1.6 \mathrm{nM}$ streptavidin) with the threshold voltage shift that the device experiences after being subjected to stabilization procedure for an hour $(\approx 1$ $\mathrm{mV} / \mathrm{h}$ ), it is concluded that the recorded threshold shifts for different concentrations of streptavidin can solely be attributed to biotin-streptavidin binding event taking place on the surface of F-gate, and not to any instability of the channel. A threshold voltage shift of $87 \mathrm{mV}$ is measured upon exposure of the sensing gate to $1.6 \mu \mathrm{M}$ streptavidin (Figure 3c), while $34 \%$ decrease in normalized current change is recorded for the same concentration of the target protein (Figure S3). The dose curve obtained based on the threshold voltage shift can be fitted with a Langmuir adsorption isotherm model being presented as a dashed line in Figure 3c.

The selectivity of the sensor was evaluated by recording its response toward bovine serum albumin (BSA), which has a comparable molecular weight with respect to streptavidin ( 60 $\mathrm{kDa}$ ), as biotin does not have a selective affinity to BSA. Figure $3 \mathrm{~d}$ presents the transfer characteristic curve recorded for biotin-immobilized F-gate in presence of $1.6 \mu \mathrm{M}$ BSA, compared to the one obtained for an exposure to $1.6 \mu \mathrm{M}$ streptavidin. An average threshold voltage shift of $5.4 \mathrm{mV}$ (three physically different gates where tested) is recorded in response to $1.6 \mu \mathrm{M}$ BSA, which is negligible compared to threshold voltage shift of $87 \pm 5.7 \mathrm{mV}$ obtained for $1.6 \mu \mathrm{M}$ streptavidin. This experiment successfully rules out the possibility of nonspecific absorption at the surface of the sensing gate, ensuring the device selectivity toward streptavidin.

To gain a better insight into the device potentiometric sensing mechanism, one can consider the biotin-terminated biorecognition layer immobilized on the F-gate as an ion-selective 
membrane. ${ }^{6}$ As the excess interfacial charge of biotin-terminated layer is screened by counterions in water, an electrochemical potential drop is created which is a quantitative function of the concentrations of the biotin, streptavidin and the resulting binding complex according to Donnan equation: ${ }^{6}$

$\Delta \phi=\frac{k_{B} T}{e}\left(\sinh ^{-1} \frac{W[R B]}{2 Y[B]}-\sinh ^{-1} \frac{X[R]}{2 Y[B]}\right)$

Where $k_{B}$ is Boltzmann constant and $T$ the temperature, $\Delta \phi$ is the electrochemical potential drop, $R$ is biotin that carries charge $X, B$ is streptavidin that possess charge $Y$, and $R B$ is the resulting binding complex that carries charge $W$. Such a potential drop at F-gate/electrolyte interface has to be compensated by applying a more negative $V_{\mathrm{g}}$ to achieve a given value of current, meaning that the device demonstrates a negative shift in its threshold voltage.

a

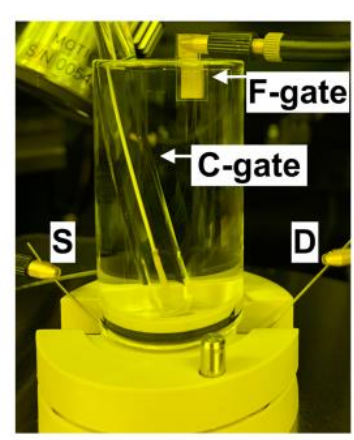

C

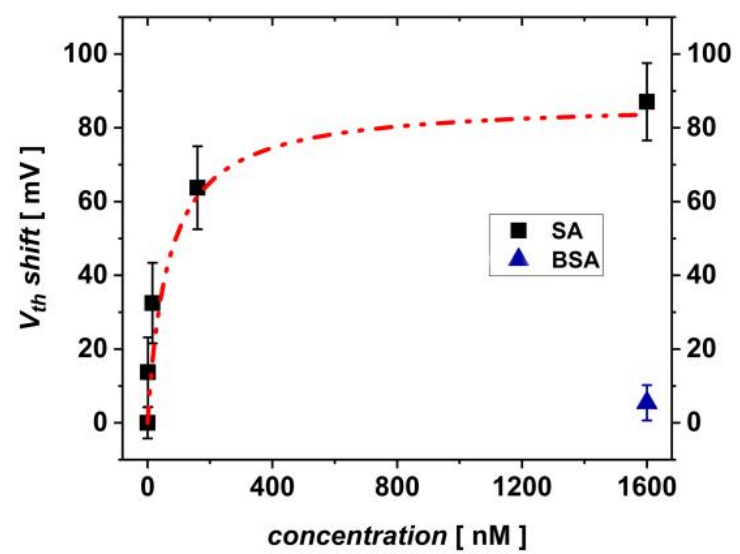

b
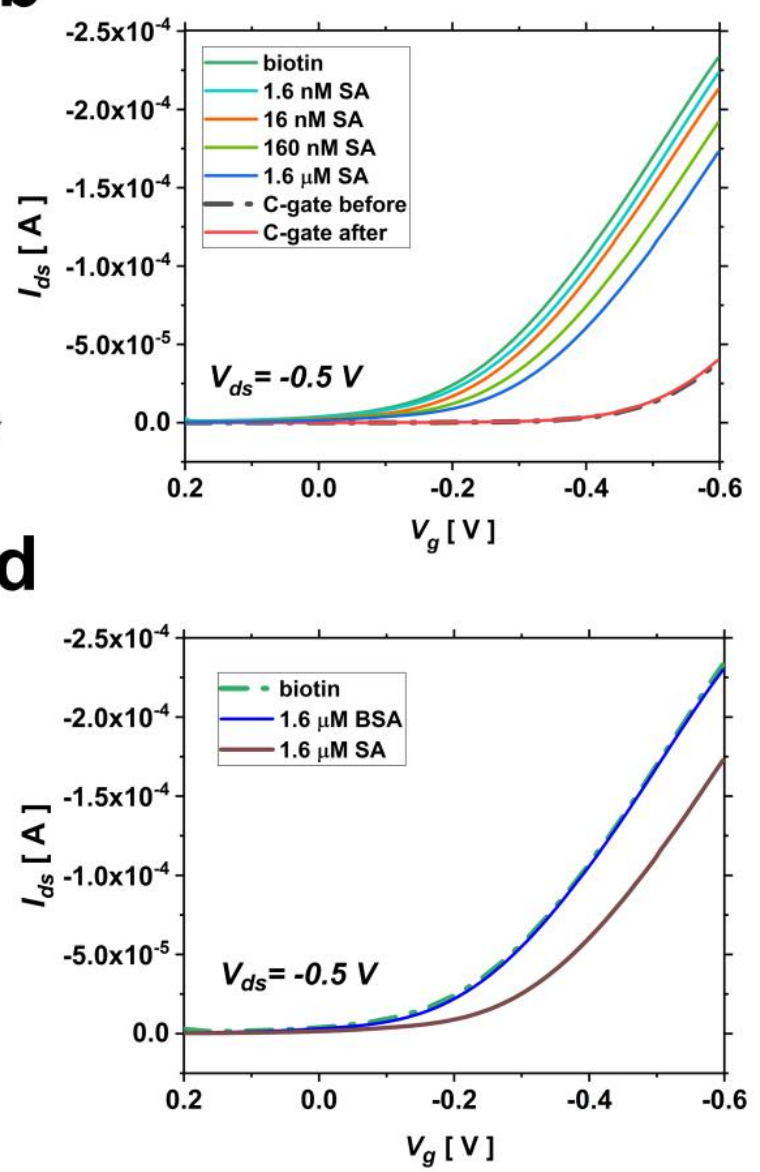

Figure 3. (a) Biosensing setup which uses two gates: F-gate for biofunctionalization and C-gate $(\mathrm{Ag} / \mathrm{AgCl})$ for controlling the stability of the channel while being exposed to the DI water; top right: detail of the F-gate; bottom right: schematic illustration of the biotin-terminated SAM immobilized on F-gate. (b) Transfer characteristic curves obtained for F-gate exposed to different concentrations of streptavidin. (c) Calibration curves acquired for threshold voltage shift, together with a Langmuir 
adsorption isotherm fit (dashed line); blue triangle symbol shows the device response to 1.6 $\mu \mathrm{M}$ BSA. (d) Negative control test comparing the transfer curves recorded for a non-exposed sensing gate denoted as "biotin", a sensing gate exposed to a $1.6 \mu \mathrm{M}$ streptavidin solution and to a $1.6 \mu \mathrm{M}$ BSA solution.

Our finding is consistent with previous reports where a threshold voltage shift was evidenced as the main consequence of the binding event. , $^{4,8,21,38}$

The extraction of the transconductance of the device for different concentrations of streptavidin (Figure S5a) shows the presence of a noticeable shift toward negative potentials due to threshold voltage shift of the device. Maximum transconductance values $\left(g_{m}^{\max }\right)$ for different concentrations of streptavidin were extracted from Figure S5a. By calculating the normalized maximum transconductance change $\left(\Delta g_{m}^{\max } / g_{m_{0}}^{\max }\right)$ for different protein concentrations, a decrease of $3.6 \%$ is recorded for $1.6 \mu \mathrm{M}$ concentration (Figure S5b). This is a relatively small change when compared to $34 \%$ decrease in $\Delta I / I_{0}$ measured for the same concentration of the protein (Figure S3). This indicates that the overall capacitance of the device has only slightly changed, assuming channel's mobility to be unaffected by protein binding. This is explained by our device design characteristics where the gate surface area is 100 times larger than the active area of the device resulting in much larger capacitance at gate/electrolyte interface. In this case the drain current remains unaffected by the variation in the capacitance at gate/electrolyte interface, where the biorecognition layer is immobilized.

Finally, the theoretical LOD was calculated from the dose curve based on the threshold voltage shift presented in Figure 3c. The LOD was measured as the concentration of streptavidin that corresponds to a response of $\left(V_{t h}\right)_{\text {mean }} \pm k \sigma$, where $\left(V_{t h}\right)_{\text {mean }}$ is the average value of the blank signal, $\sigma$ is the standard deviation of the blank sample and $k$ is a numerical factor selected based on the confidence level needed. IUPAC suggests a value of $k=3$ to be acceptable since the probability of a false positive would be less than $1 \% .{ }^{21}$ The standard deviation of the blank measurement (biotin functionalized F-gate) was calculated as $4.23 \mathrm{mV}$, which returns a theoretical LOD of $1.47 \mathrm{nM}$ based on Langmuir fit (dashed line) presented in Figure 3c. Comparing to an only report on sensing streptavidin by an EGOFET, ${ }^{15}$ our device showed 7 times lower LOD while having almost the same range.

\section{CONCLUSION}

We have proposed a water gated FET based on cost-effective inkjet-printed random networks of polymer-wrapped s-SWCNTs as a candidate for future biosensors. Our EG-CNTFET requires only an hour of device conditioning in water, a much shorter time with respect to analogue polymer-based devices exploited for highly sensitive biosensors. After conditioning, 
the water gated device demonstrates excellent stability, with a $V_{\text {th }}$ shift of $1 \mathrm{mV} / \mathrm{h}$ and $\Delta I / I_{0}$ decrease rate of $0.002 \mathrm{~h}^{-1}$. When gated through water, the devices show a normalized transconductance ( $g_{\mathrm{m}}$ divided by $W / L$ ) of $0.44 \mu \mathrm{S}$, being at least one order of magnitude higher compared to its EGOFET counterparts.

As a proof-of-principle, we also demonstrate the potential applicability of our EG-CNTFET in biosensing, by characterizing the well-known biotin-streptavidin binding event through immobilization of biotin on the transistor gate. The biosensor correctly operates in the tested range of $1.6 \mathrm{nM}$ to $1.6 \mu \mathrm{M}$ of streptavidin, has an estimated LOD of $1.47 \mathrm{nM}$ and it shows no response to BSA in a negative control experiment, indicating selectivity. Having validated the compatibility of the device with such biosensing scheme, our work paves the way for the future exploration of the use of such stable and performing platform with a plethora of recognition strategies, including protein and genomic based ones, and of the adoption of advanced biofunctionalization protocols, with the potential to provide a very high sensitivity.

Overall, our EG-CNTFET shares the benefits of cost-effective and large-area processing of polymer EGOFETS, while being characterized by a higher normalized transconductance, a shorter conditioning time and excellent stability to operation in water. For such reasons we believe it is a powerful candidate for applications in the bioelectronics field, offering a reliable platform for fast response diagnostic tools.

\section{Funding Sources}

F. A. V. and M. C. acknowledge the financial support under the European Union's Horizon 2020 research and innovation programme - "Electronic Smart Systems - SiMBiT: Single molecule bio-electronic smart system array for clinical testing”, grant agreement 824946.

\section{Acknowledgements}

The fabrication of the devices was partially carried out at PoliFab, the micro and nanotechnology center of the Politecnico di Milano. Authors want to thank Dr Bobur Mirkhaydarov and Fabio Bersano for helping with device fabrication.

\section{Supporting Information}

Description of fabrication procedure of P3HT based EGOFET; extracted current values at -0.8 $\mathrm{V}$ from consecutive transfer curves acquired for P3HT based EGOFET for $65 \mathrm{~h}$; calibration curve based on normalized current change at $-0.5 \mathrm{~V}$ for EG-CNTFET; typical transfer curves 
for bare Au and Cys-SAM functionalized Au electrodes; transconductance curves for different concentrations of streptavidin together with titration curve based on extracted maximum transconductance values. 


\section{REFERENCES}

1. Bergveld, P. Thirty years of ISFETOLOGY: What happened in the past 30 years and what may happen in the next 30 years. Sensors Actuators, B Chem. 88, 1-20 (2003).

2. White, S. P., Frisbie, C. D. \& Dorfman, K. D. Detection and Sourcing of Gluten in Grain with Multiple Floating-Gate Transistor Biosensors. ACS Sensors 3, 395-402 (2018).

3. Cosseddu, P., Viola, F., Lai, S., Raffo, L., Seminara, L., Pinna, L., Valle, M., Dahiya, R. \& Bonfiglio, A. Tactile sensors with integrated piezoelectric polymer and low voltage organic thin-film transistors. in IEEE SENSORS 2014 Proceedings vols 2014Decem 1734-1736 (IEEE, 2014).

4. Macchia, E., Manoli, K., Holzer, B., Di Franco, C., Ghittorelli, M., Torricelli, F., Alberga, D., Mangiatordi, G. F., Palazzo, G., Scamarcio, G. \& Torsi, L. Singlemolecule detection with a millimetre-sized transistor. Nat. Commun. 9, 3223 (2018).

5. Romele, P., Ghittorelli, M., Kovács-Vajna, Z. M. \& Torricelli, F. Ion buffering and interface charge enable high performance electronics with organic electrochemical transistors. Nat. Commun. 10, 1-11 (2019).

6. Picca, R. A., Manoli, K., Macchia, E., Sarcina, L., Di Franco, C., Cioffi, N., Blasi, D., Österbacka, R., Torricelli, F., Scamarcio, G. \& Torsi, L. Ultimately Sensitive Organic Bioelectronic Transistor Sensors by Materials and Device Structure Design. Adv. Funct. Mater. 30, 1-23 (2020).

7. Casalini, S., Leonardi, F., Cramer, T. \& Biscarini, F. Organic field-effect transistor for label-free dopamine sensing. Org. Electron. 14, 156-163 (2013).

8. Lin, P., Luo, X., Hsing, I. M. \& Yan, F. Organic electrochemical transistors integrated in flexible microfluidic systems and used for label-free DNA sensing. Adv. Mater. 23, 4035-4040 (2011).

9. Mulla, M. Y., Tuccori, E., Magliulo, M., Lattanzi, G., Palazzo, G., Persaud, K. \& Torsi, L. Capacitance-modulated transistor detects odorant binding protein chiral interactions. Nat. Commun. 6, 1-9 (2015).

10. Dey, A., Singh, A., Dutta, D., Ghosh, S. S. \& Iyer, P. K. Rapid and label-free bacteria detection using a hybrid tri-layer dielectric integrated n-type organic field effect transistor. J. Mater. Chem. A 7, 18330-18337 (2019).

11. Berto, M., Diacci, C., D’Agata, R., Pinti, M., Bianchini, E., Lauro, M. D., Casalini, S., Cossarizza, A., Berggren, M., Simon, D., Biscarini, F. \& Bortolotti, C. A. EGOFET Peptide Aptasensor for Label-Free Detection of Inflammatory Cytokines in Complex Fluids. Adv. Biosyst. 2, (2018).

12. Park, M.-H., Han, D., Chand, R., Lee, D.-H. \& Kim, Y.-S. Mechanism of Label-Free DNA Detection Using the Floating Electrode on Pentacene Thin Film Transistor. $J$. Phys. Chem. C 120, 4854-4859 (2016).

13. Viola, F. A., Brigante, B., Colpani, P., Dell'Erba, G., Mattoli, V., Natali, D. \& Caironi, M. A 13.56 MHz Rectifier Based on Fully Inkjet Printed Organic Diodes. Adv. Mater. 32, 1-7 (2020).

14. Stucchi, E., Scaccabarozzi, A. D., Viola, F. A. \& Caironi, M. Ultraflexible all-organic complementary transistors and inverters based on printed polymers. J. Mater. Chem. C 8, 15331-15338 (2020).

15. Magliulo, M., Mallardi, A., Mulla, M. Y., Cotrone, S., Pistillo, B. R., Favia, P., Vikholm-Lundin, I., Palazzo, G. \& Torsi, L. Electrolyte-gated organic field-effect transistor sensors based on supported biotinylated phospholipid bilayer. Adv. Mater. 25, 2090-2094 (2013).

16. Macchia, E., Manoli, K., Holzer, B., Di Franco, C., Picca, R. A., Cioffi, N., Scamarcio, 
G., Palazzo, G. \& Torsi, L. Selective single-molecule analytical detection of C-reactive protein in saliva with an organic transistor. Anal. Bioanal. Chem. 411, 4899-4908 (2019).

17. Seshadri, P., Manoli, K., Schneiderhan-Marra, N., Anthes, U., Wierzchowiec, P., Bonrad, K., Di Franco, C. \& Torsi, L. Low-picomolar, label-free procalcitonin analytical detection with an electrolyte-gated organic field-effect transistor based electronic immunosensor. Biosens. Bioelectron. 104, 113-119 (2018).

18. Blasi, D., Viola, F., Modena, F., Luukkonen, A., MacChia, E., Picca, R. A., Gounani, Z., Tewari, A., Österbacka, R., Caironi, M., Kovacs Vajna, Z. M., Scamarcio, G., Torricelli, F. \& Torsi, L. Printed, cost-effective and stable poly(3-hexylthiophene) electrolyte-gated field-effect transistors. J. Mater. Chem. C 8, 15312-15321 (2020).

19. Ricci, S., Casalini, S., Parkula, V., Selvaraj, M., Saygin, G. D., Greco, P., Biscarini, F. \& Mas-Torrent, M. Label-free immunodetection of $\alpha$-synuclein by using a microfluidics coplanar electrolyte-gated organic field-effect transistor. Biosens. Bioelectron. 167, 112433 (2020).

20. MacChia, E., Tiwari, A., Manoli, K., Holzer, B., Ditaranto, N., Picca, R. A., Cioffi, N., Di Franco, C., Scamarcio, G., Palazzo, G. \& Torsi, L. Label-Free and Selective SingleMolecule Bioelectronic Sensing with a Millimeter-Wide Self-Assembled Monolayer of Anti-Immunoglobulins. Chem. Mater. 31, 6476-6483 (2019).

21. Macchia, E., Manoli, K., Di Franco, C., Picca, R. A., Österbacka, R., Palazzo, G., Torricelli, F., Scamarcio, G. \& Torsi, L. Organic Field-Effect Transistor Platform for Label-Free, Single-Molecule Detection of Genomic Biomarkers. ACS Sensors $\mathbf{5}$, 1822-1830 (2020).

22. Sailapu, S. K., Macchia, E., Merino-Jimenez, I., Esquivel, J. P., Sarcina, L., Scamarcio, G., Minteer, S. D., Torsi, L. \& Sabaté, N. Standalone operation of an EGOFET for ultra-sensitive detection of HIV. Biosens. Bioelectron. 156, 1-7 (2020).

23. Picca, R. A., Manoli, K., Macchia, E., Tricase, A., Di Franco, C., Scamarcio, G., Cioffi, N. \& Torsi, L. A Study on the Stability of Water-Gated Organic Field-EffectTransistors Based on a Commercial p-Type Polymer. Front. Chem. 7, 1-10 (2019).

24. Zhang, Q., Leonardi, F., Casalini, S., Temiño, I. \& Mas-Torrent, M. High performing solution-coated electrolyte-gated organic field-effect transistors for aqueous media operation. Sci. Rep. 6, 1-10 (2016).

25. Bhatt, V. D., Joshi, S., Becherer, M. \& Lugli, P. Flexible, Low-Cost Sensor Based on Electrolyte Gated Carbon Nanotube Field Effect Transistor for Organo-Phosphate Detection. Sensors (Basel). 17, 1147 (2017).

26. Okuda, S., Okamoto, S., Ohno, Y., Maehashi, K., Inoue, K. \& Matsumoto, K. Horizontally aligned carbon nanotubes on a quartz substrate for chemical and biological sensing. J. Phys. Chem. C 116, 19490-19495 (2012).

27. Lerner, M. B., Dailey, J., Goldsmith, B. R., Brisson, D. \& Charlie Johnson, A. T. Detecting Lyme disease using antibody-functionalized single-walled carbon nanotube transistors. Biosens. Bioelectron. 45, 163-167 (2013).

28. Star, A., Gabriel, J. C. P., Bradley, K. \& Grüner, G. Electronic detection of specific protein binding using nanotube FET devices. Nano Lett. 3, 459-463 (2003).

29. Filipiak, M. S., Rother, M., Andoy, N. M., Knudsen, A. C., Grimm, S., Bachran, C., Swee, L. K., Zaumseil, J. \& Tarasov, A. Highly sensitive, selective and label-free protein detection in physiological solutions using carbon nanotube transistors with nanobody receptors. Sensors Actuators, B Chem. 255, 1507-1516 (2018).

30. Rother, M., Kruse, A., Brohmann, M., Matthiesen, M., Grieger, S., Higgins, T. M. \& Zaumseil, J. Vertical electrolyte-gated transistors based on printed single-walled carbon nanotubes. ACS Appl. Nano Mater. 1, 3616-3624 (2018). 
31. Graf, A., Zakharko, Y., Schieß1, S. P., Backes, C., Pfohl, M., Flavel, B. S. \& Zaumseil, J. Large scale, selective dispersion of long single-walled carbon nanotubes with high photoluminescence quantum yield by shear force mixing. Carbon N. Y. 105, 593-599 (2016).

32. Zorn, N. F., Scuratti, F., Berger, F. J., Perinot, A., Heimfarth, D., Caironi, M. \& Zaumseil, J. Probing Mobile Charge Carriers in Semiconducting Carbon Nanotube Networks by Charge Modulation Spectroscopy. ACS Nano 14, 2412-2423 (2020).

33. Scuratti, F., Bonacchini, G. E., Bossio, C., Salazar-Rios, J. M., Talsma, W., Loi, M. A., Antognazza, M. R. \& Caironi, M. Real-Time Monitoring of Cellular Cultures with Electrolyte-Gated Carbon Nanotube Transistors. ACS Appl. Mater. Interfaces 11, 37966-37972 (2019).

34. Seifert, M., Rinke, M. T. \& Galla, H. J. Characterization of streptavidin binding to biotinylated, binary self-assembled thiol monolayers - Influence of component ratio and solvent. Langmuir 26, 6386-6393 (2010).

35. Lowe, B. M., Sun, K., Zeimpekis, I., Skylaris, C. K. \& Green, N. G. Field-effect sensors-from $\mathrm{pH}$ sensing to biosensing: Sensitivity enhancement using streptavidinbiotin as a model system. Analyst 142, 4173-4200 (2017).

36. King, S. J., Miller, H. R. P., Bloch, J., Jarrett, E., Bazin, H., Cs, T. A. V, Macqueen, G., Siegel, S., The, C., Ii, R., The, B., The, G. \& Gabella, G. Structural Origins of High-Affinity Biotin Binding. 391, 85-88 (1987).

37. Rother, M., Brohmann, M., Yang, S., Grimm, S. B., Schieß1, S. P., Graf, A. \& Zaumseil, J. Aerosol-Jet Printing of Polymer-Sorted (6,5) Carbon Nanotubes for FieldEffect Transistors with High Reproducibility. Adv. Electron. Mater. 3, 1-9 (2017).

38. Berto, M., Casalini, S., Di Lauro, M., Marasso, S. L., Cocuzza, M., Perrone, D., Pinti, M., Cossarizza, A., Pirri, C. F., Simon, D. T., Berggren, M., Zerbetto, F., Bortolotti, C. A. \& Biscarini, F. Biorecognition in organic field effect transistors biosensors: The role of the density of states of the organic semiconductor. Anal. Chem. 88, 1233012338 (2016).

39. Kergoat, L., Herlogsson, L., Braga, D., Piro, B., Pham, M. C., Crispin, X., Berggren, M. \& Horowitz, G. A water-gate organic field-effect transistor. Adv. Mater. 22, 25652569 (2010). 


\section{For table of contents only}

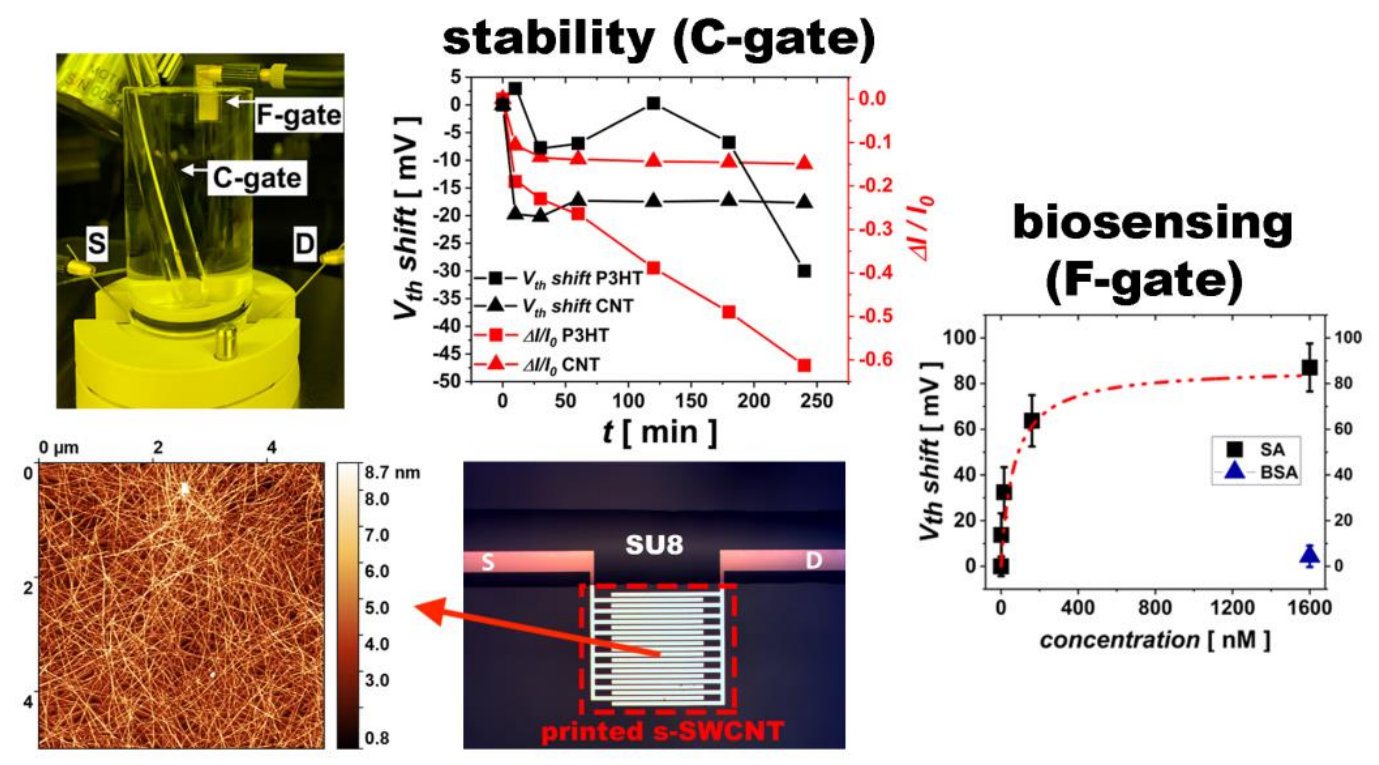

